\title{
Attention-based Design and Selective Exposure Amid COVID-19 Misinformation Sharing
}

\author{
Zaid Amin ${ }^{1,2}$, Nazlena Mohamad Ali*1, Alan F Smeaton ${ }^{3}$ \\ ${ }^{1}$ Institute of IR4.0 (IIR4.0), Universiti Kebangsaan Malaysia, Malaysia \\ ${ }^{2}$ Faculty of Informatics Engineering, Universitas Bina Darma, Indonesia \\ ${ }^{3}$ INSIGHT: Centre for Data Analytics, Dublin City University, Dublin 9, Ireland

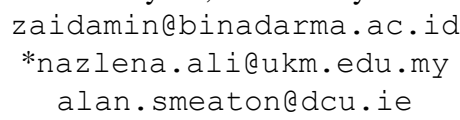

Abstract. One of the significant limitations in human behaviour when receiving online information is our lack of visual cognitive abilities, the ability to pay greater attention in a short time. The question arises about how we handle online messages, which contain and send people with the same associated interests as ourselves, regarding social influences and individual beliefs. This study aims to provide some insight into misinformation sharing. The availability of enormous amounts of COVID-19 information makes the selectivity of messages likely limited by the distortion of perceptions in the communicating environment. It is also in line with the fact that human attention is essentially limited and depends on the conditions and tasks at hand. To understand this phenomenon, we proposed a Tuning Attention Model (TAM). The model proposes tuning and intervene in a user's attention behaviour by incorporating an attention-based design when users decide to share COVID-19 misinformation. In pilot study results, we found that attention behaviour negatively correlated with misinformation sharing behaviour. The results justify that when attention behaviour increased, misinformation sharing behaviour will decrease. We suggest an attention-based design approached on social media application's that could intervene in user attention and avoid selective exposure caused by the spread of COVID-19 misinformation. The study expected to produce continuous knowledge leading to non-coercive handling of sharing COVID-19 misinformation behaviour and laying the basis for overcoming misinformation issues.

Keywords: Attention, design, selective exposure, COVID-19, misinformation sharing, user interfaces 


\section{Introduction}

Since the advent of online messages, our internal and external environments have been flooded with rising amounts of information. According to Cisco (2018), "Global IP traffic is expected to reach 396 Exabyte per month by 2022, up from 122 Exabyte per month in 2017 . There will be 4.8 billion internet users by 2022. That's up from 3.4 billion in 2017 or 45 percent of the world's population". Being aware of the incredible explosion of information today in online media makes us realize that it is significantly inversely comparable to our ability to access it. It is merely because our attention remains very limited. As humans, we have limited cognitive skills in processing and understanding information obtained from the surrounding environment. Therefore, in the early stages of every task-oriented, it is essential for those focusing on designing $\mathrm{Hu}$ man-Computer Interaction (HCI) to examine characteristics of attention and their interactive association with action planning (Salihan, Nazlena, \& Masnizah, 2017).

Understanding the value or quality of information is also vital to how users should consume information for constructive purposes or have a detrimental impact on individuals or social structures. Amid the increasing burden of the COVID-19 pandemic, there are parallel emergencies that need to be tackled simultaneously - the rise of counterfeit drugs, fake news, and misinformation on treatment around COVID-19. Credible sources of information from health experts are also the key to justifying health professionals' suggestions with the skills and training necessary to fight this emergency. Essentially, they can be a source of accurate and reliable information relevant to the public or other associate health professionals, thereby reducing the spread of misinformation on the treatment of COVID-19. Ideal conditions can achieve by presenting accurate and reliable information based on relevant health authorities and professional associations recommendations to ensure that the public is not affected by other exposure factors.

COVID-19 is not only a global pandemic and has a multidimensional effect. According to the WHO, it is also an "infodemic", highlighting the immediate social problems arising from a large amount of misinformation and fake news circulating about COVID-19 (Laato et al., 2020). Numerous issues of COVID-19 misinformation are relatively found in social media, which plays an essential role in the spread of misinformation (Allcott and Gentzkow, 2017). It also raises questions regarding what platforms can prevent fake news spread (Figueira and Oliveira, 2017). During the COVID19 pandemic, clear communication about the severity of the situation and suggested medical standards are needed to ensure people take the right action and do not suffer unnecessary anxiety (Farooq et al., 2020). The abundance of unclear, ambiguous, and incorrect information during COVID-19 leads to information overload and accelerated health anxiety (Laato et al., 2020). Although sufficient research has shown that people exhibit confirmation bias related to selective exposure on social media, the lack of research trying to untangle the several effects of selective exposure and sharing in the context of COVID-19 is still urgently needed. 
This study aims to produce a model that can describe the selective phenomenon amid COVID-19 misinformation and proposed attention-based design, which is expected to intervene in the attention of user interactions in the behaviour of sharing misinformation. As well as modelling the psychological dimensions occurs with attention-based design approach solutions.

The paper is structured as follows. The first part contains an introduction that briefly describes the phenomenon of selective exposure in COVID-19 misinformation. The second part includes a background on the existing literature on attention-based design, selective exposure, and its relationship to the spread of misinformation about COVID19. The third section contains the material and method applied. The fourth section contains the result's main findings, and the fifth part includes a discussion of the implications and the sustainability of future research.

\section{Background}

\subsection{Attention-based design}

Designing a system that can intervene in user attention is a significant challenge for research in HCI and cognitive techniques. Attention interface is one crucial part of the human and machine (computer) interaction process, which explicitly focuses on human attention as an essential input for computers. One of the most dominant modalities is to increase user attention with interface application design through a visual approach. Visual is the dominant modality for information transfer in HCI (Spence, Lloyd, McGlone, Nichols, \& Driver, 2000). One example is the concept of selective visual attention, which is closely related to increasing attention behaviour and can increase the certainty of choice in human decision making and goal-directed behaviour in facing an efficient task when viewed from time consumption (Zizlsperger et al., 2012; Chelazzi, 2012). Gaining insight into the user's visual attention is very important to obtain information about the influence of brand or product sales in consumer decision making (Pieters and Warlop, 1999).

Because humans have limited cognitive resources and capacities, we struggle with information overload in today's information-rich society (Edmunds \& Morris, 2000). Deciding which material to access or attend is a challenge for individuals (Dukas, 2002), but at the same time, offers an exploratory topic of interest to researchers. Such information overload has also manifested itself in academia and other fields, which has developed into a "battle of attention" (Torgler \& Piatti, 2013). Readers face the tradeoffs of what to read and, therefore, allocate their attention to neglecting other stimuli. In general, attention is explored in various fields, such as cognitive science, neuroscience, sociology, or primatology (Lanham, 2006). It has been defined (collectively) as "psychological and neural mechanisms that mediate perceptual selectivity" (Yantis, 2000). 
Previous research suggests that designers should view attention as a dynamic process when designing attention management views, particularly regarding handling COVID19 misinformation sharing behaviour. Visual display events that temporarily precede the contextual content of a current message may need to be considered for further investigation. A developer can make several technical approaches. First, to use a zoomlens metaphor, previous views may have affected the user's current attention 'zoom setting'. Second, the last state view may have resulted in the user shaping his current area of concern to a specific object shape. Third, there may be a negative priming effect of previous stimuli that the user is actively trying to ignore. Lee \& Choo (2013) reviewed how attention works using appropriate metaphors. According to the spotlight metaphor, attention can be characterized as an internal radiance illuminating the location where an object is placed and clearly enhances the user's focus (Posner, Snyder, \& Davidson 1980). Hodas and Lerman (2012), mention the attentiveness factor is inherently the property of individual users and is limited for each task and situation. Social media designers would influence users through the user interface's design choices. Interface design can manipulate user visibility to maximize user attention when consuming COVID-19 information on social media applications.

\subsection{Selective exposure}

Attention to COVID-19 continues to grow on Twitter and possibly other platforms as well. People tend to care about the news that exposures them personally (shares their own beliefs), making sense that relevant conversations will develop as the pandemic continues to involve more people on a personal level. Likewise, attention is focused on the countries hardest hit by the disease, demonstrating that attention, discussion, and information sharing have the most significant impact on those most affected (Singh et al., 2020).

However, at the end of February 2020, the comparative flu myth and the misinformation of home remedies for COVID-19 appeared almost as frequently in the data collected by Singh et al. (2020), although there is some evidence to suggest that the comparative flu myth may have declined by the end of their data collection. In mid-March, the myths about heat kill COVID-19 and vaccine development also emerged from time to time. Users' selective exposure maintained their relative position compared to other myths and tended to reflect only topic's conversations. It is also important to note that tweets that counter or debunk certain COVID-19 myths are also likely to be part of an identified tweets pool. Almost everyone agrees that "prevention is better to care for". However, the critical prevention process is not only the responsibility of stakeholders (government, health agencies, and experts). Furthermore, the fundamental prevention of the infodemic problem is how to reduce the exposure that occurs continuously to someone's knowledge and belief. If it is not mediated, it will quickly "crystallize" in behaviour and create new social problems in addressing COVID-19 treatment. 
Besides, as seen in the prevention of COVID-19, according to Singh et al. (2020), recipients and stakeholders can lose patience with prevention because evaluations that are not obtained immediately provide results. Although other evaluation types (e.g., formative) are useful, stakeholders (e.g., leaders, political figures) may intervene to fix problems quickly. However, as evidenced by the rushed attention to developing a vaccine for COVID-19, infectious disease scientists remind us that development will take a long time, require collaboration across the scientific community, and incur considerable costs before it becomes robust and secure (Corey et al., 2020).

A significant impact that continues to occur is that selective exposure can lead to an echo chamber's emergence on social media. A group of like-minded people working together to frame and reinforce a shared narrative, thus facilitating fake news and the general flow of COVID-19 misinformation. In both cases, the main driver of selective exposure is interested in a topic, where people prefer information that matches their interests while avoiding off-topic information. The understanding of interest as a driver of attention is also at the root of selective exposure theory. Based on cognitive dissonance theory, selective exposure is defined as "preference information consistent with previously held beliefs along with avoidance of information contrary to such beliefs" (Graf \& Aday, 2008).

\section{$3 \quad$ Material and methods}

The method used in this study is carried out, starting with the determination of sampling and questionnaire design. The data collection technique in this study used nonprobability sampling with a purposive sampling method approach. The instrument used was an online questionnaire that provides questions to determine the user's answers (n $=112$ ) about the relationship of attention behaviour in sharing misinformation on social media.

The questions consist of demographic information, gender, profession, age, education, and frequently used social media platforms. Respondents were asked to choose the most appropriate item (5 Likert scales). One example of questionnaire materials is $\mathrm{s}$ shown in Figure 1. This question is related to whether they would share information if there were information about the application of the rubella vaccine in children, and the narrative of this application can cause an adverse effect. This question also investigates whether a selective exposure factor occurs when the user decides to share or not share the question given. 


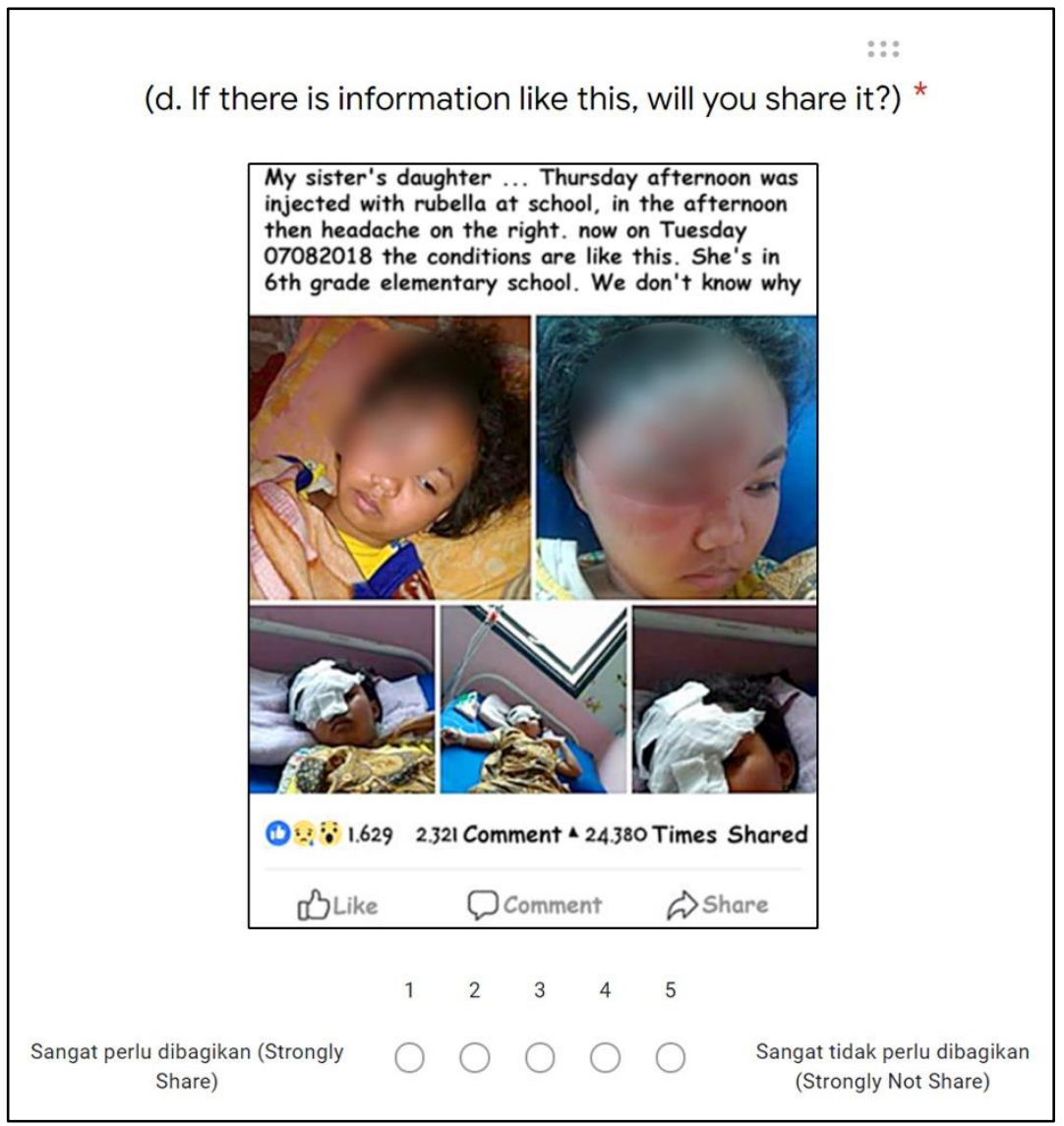

Fig 1. An example of the questionnaire materials regarding the harmful effects of applying the rubella vaccine to children

\section{Results}

The pilot study findings in a questionnaire show selective exposure happened when users asked to share or not share information circulating on social media. These findings are very relevant to the issue of medical knowledge in dealing with sharing COVID-19 misinformation. The following are some of the results that we highlight concerning the pilot studies we have conducted. 


\subsection{Questionnaire Results}

We collected data from 112 respondents from October 1, 2018, to November 30, 2018 (8 weeks). Demographic data collected consists of numerous sections, namely gender, age group, recent education, occupation, and cyberspace most regularly used (see Table 1). The results show that the sample contains slightly more women $(55.4 \%)$. The origin of the largest participating country is Indonesia at $92.9 \%$. The most widely used social media platform is WhatsApp at $86.6 \%$, and the age range of most participants is $30-39$ years at $39.3 \%$.

Table 1. Respondent's demographics.

\begin{tabular}{|c|c|c|c|c|c|}
\hline Demograp & ic Data & Number & Demographic & & $\begin{array}{l}\text { Number } \\
\text { Percent- }\end{array}$ \\
\hline Gender & Male & $44.6 \%$ & $\begin{array}{l}\text { Country of } \\
\text { origin }\end{array}$ & Indonesia & $92.9 \%$ \\
\hline & Female & $55.4 \%$ & & Malaysia & $7.1 \%$ \\
\hline Age & $<17$ years & $12.5 \%$ & $\begin{array}{l}\text { The most used } \\
\text { cyberspace }\end{array}$ & Twitter & $11.6 \%$ \\
\hline & $18-20$ years & $2.7 \%$ & & WhatsApp & $86.6 \%$ \\
\hline & $21-29$ years & $33 \%$ & & Facebook & $50 \%$ \\
\hline & $30-39$ years & $39.3 \%$ & & Instagram & $55.4 \%$ \\
\hline & $40-49$ years & $8 \%$ & & YouTube & $24.1 \%$ \\
\hline & $50-69$ years & $4.5 \%$ & & & \\
\hline & $60>$ years & $2.7 \%$ & & & \\
\hline $\begin{array}{l}\text { Highest } \\
\text { Education }\end{array}$ & $\begin{array}{l}\text { Less than high } \\
\text { school degree }\end{array}$ & $12.5 \%$ & & & \\
\hline & $\begin{array}{l}\text { High school de- } \\
\text { gree }\end{array}$ & $15.2 \%$ & & & \\
\hline & Diploma degree & $3.6 \%$ & & & \\
\hline & Bachelor degree & $40.2 \%$ & & & \\
\hline & Master degree & $28.6 \%$ & & & \\
\hline & $\begin{array}{l}\text { Postgraduare de- } \\
\text { gree }\end{array}$ & $12.5 \%$ & & & \\
\hline
\end{tabular}

The questionnaire results also show that $34 \%$ of 112 respondents agree that users easily share misinformation without reading the content first (especially when dealing with their exposures, including beliefs and social influences). Approximately $75 \%$ of respondents agree that sharing misinformation with attentive behaviour is essential. Previous research has confirmed that quality is not a mandatory requirement for online virality (Weng, 2012). The results we obtained in examining selective exposure factors clearly show that the top element is an epistemic belief within one's internal self. This 
epistemic belief factor becomes affected when social influences factors predominate. These results are consistent with justification from Chua et al. (2017), who mentions that epistemic belief significantly affected users' decisions to share online health rumours. The question about belief factors in sharing misinformation shows that $78 \%$ of respondents share information because they want to share their beliefs with others. The majority of respondents share information on social media because they want to help and share the knowledge they believe in others. It is also stated by Garrett \& Weeks (2017), which is that individuals are inclined to think misperceptions, and this exposure/bias rises with opportunity (e.g., time to think) and with the ability (e.g., cognitive resources). Furthermore, we find that selective exposure to social influences factors emerge in second place. Still, these two states are also influenced by how long it takes the user to pay more attention to the information obtained.

One of the material's contexts was medical information containing rumours spread on social media about vaccinating children's harmful impacts. This question shows that 112 participants dominantly answered doubtful (neutral), particularly $28.6 \%$. These results show that the participants experienced a dissonance phase, which tends to be influenced by the exposure (bias) from within them, precisely with the belief and social influence factor. These cognitive dissonance phases emerge where the user has doubts in deciding whether to share the information or not. This critical phase will "boil down" depending on how much pressure the epistemic belief and social influence factors face. This finding is consistent with McLeod's (2018) results, stating that cognitive impairment involves conflicting attitudes, beliefs, or behaviours.

\subsection{Proposed Tuning Attention Model (TAM)}

In the context of the development of a model, we construct the findings from the questionnaire results and understand related theories into an initial model that will clearly explain this phenomenon, including proposed assigning the role of attentionbased design. We built an initial concept called the Tuning Attention Model (TAM) based on the questionnaire results. This TAM model suggests the phenomena that occur, including psychological dominance and intervention tools through COVID-19 misinformation sharing by the attention-based design approach (see Figure 2). 


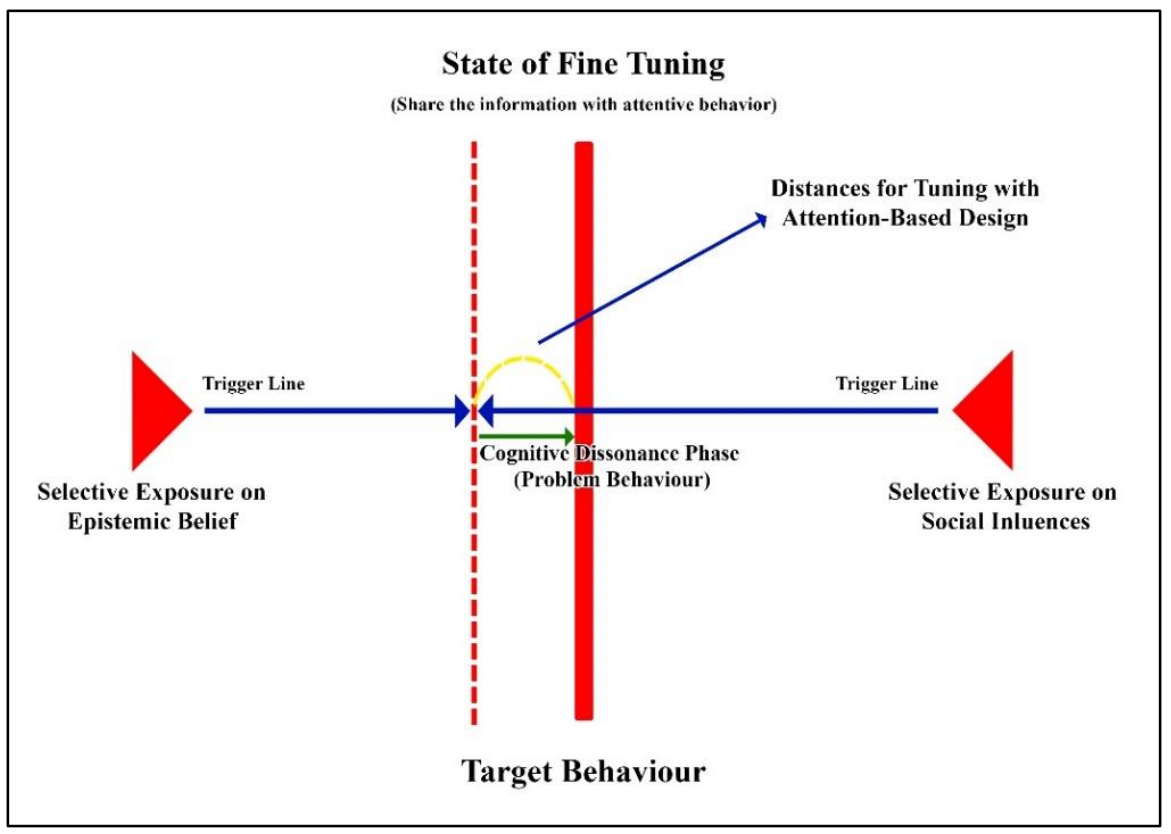

Fig. 2 Tuning Attention Model (TAM)

In the initial model (Figure 2), thick red lines indicate ideal conditions (target behaviour), where the user is at the expected level of attention. The user is exposed to higher social influence factors than the epistemic belief factor (as shown in the blue arrow as a trigger). The user tends not to have attention, and this exposure triggers user behaviour to share misinformation. This is shown in the lacking of dashed red lines.

The behavioural conditions that need to be tuned and measured are as shown in the dotted yellow line. The attention-based solution or approach used in this model is the tuning treatment (reversing the target behaviour condition). The attention-based design implies that misinformation containment policies should also emphasize behavioural interventions, like labelling and incentives to dissuade the spread of misinformation, particularly on COVID-19 misinformation. These intervention tools based on psychological understanding is significantly more essential rather than focusing exclusively on bots. These findings suggest that concerted cognitive psychological efforts are needed to be embedded on design social media applications and encourage users to flag and constructively refute misinformation (Chen et al. 2015). 


\section{Discussion}

This study's significance is a comprehensive investigation that integrates psychological aspects, which mainly become one of the HCI field's core domains. Unfortunately, very little research emphasizes the integrated approach of human cognitive psychological factors in dealing with COVID-19 misinformation sharing behaviour and in line with research by (Vosoughi et al., 2018; Bakshy et al., 2009; Cook et al., 2017), encouraging investigation on human judgment factors. These justifications imply that misinformation containment policies should also emphasize behavioural interventions, like labelling and incentives to dissuade the spread of misinformation, rather than focusing exclusively on bots.

The significants of increasing attention behaviour to influence selective exposure has been identified in this study. This study's finding indicated that attention-based design is needed to further application to COVID-19 misinformation sharing behaviour phenomena. The attention-based design also implied that a particular strategy would suggest intervening selective exposure amid COVID-19 misinformation. Research into attention-based design and how those designs would affect selective exposure on a user in sharing COVID-19 misinformation is required. Research is also needed to share useful COVID-19 information with attentive behaviour based on health sources credibility. Research that focuses on detecting COVID-19 misinformation with machine learning approaches or robotic applications is expected to be complementary when a user fails to detect COVID-19 misinformation. Research using a hybrid approach to seeing COVID-19 misinformation with a unifying machine and human collaboration will be necessary. As social beings, devices have limitations in terms of individual and social interactions.

The studies reveal that selective exposure occurs when users encounter medical information such as vaccine use in children. The selective exposure could influence user attention in handling sharing COVID-19 misinformation. By taking a practical approach to future research through attention-based design, it is expected to produce user interfaces that prioritize solving the COVID19 misinformation phenomenon in a noncoercive manner, particularly prioritizing a humanist approach.

\section{Acknowledgement}

We thank all participants in the study. The work was supported by the university research grant UKM GPK-4IR-2020-019. 


\section{References}

1. Allcott, H., \& Gentzkow, M. (2017). Social media and fake news in the 2016 election. Journal of economic perspectives, 31(2), 211-36.

2. Bakshy, E., Karrer, B., \& Adamic, L. A. (2009, July). Social influence and the diffusion of user-created content. In Proceedings of the 10th ACM conference on Electronic commerce (pp. 325-334).

3. Corey, L., Mascola, J. R., Fauci, A. S., \& Collins, F. S. (2020). A strategic approach to COVID-19 vaccine R\&D. Science, 368(6494), 948-950.

4. Cisco. (2018). Cisco Predicts More IP Traffic in the Next Five Years Than in the History of the Internet. Retrieved February 6, 2021, from https://newsroom.cisco.com/press-releasecontent?type $=$ webcontent $\&$ articleId $=1955935$

5. Chua, A. Y., \& Banerjee, S. (2017). To share or not to share: The role of epistemic belief in online health rumors. International journal of medical informatics, 108, 36-41.

6. Cook, J., Lewandowsky, S., \& Ecker, U. K. (2017). Neutralizing misinformation through inoculation: Exposing misleading argumentation techniques reduces their influence. PloS one, 12(5), e0175799.

7. Chen, X., Sin, S. C. J., Theng, Y. L., \& Lee, C. S. (2015, June). Why do social media users share misinformation?. In Proceedings of the 15th ACM/IEEE-CS joint conference on digital libraries (pp. 111-114).

8. Dukas, R. (2002). Behavioural and ecological consequences of limited attention. Philosophical Transactions of the Royal Society of London. Series B: Biological Sciences, 357(1427), 1539-1547.

9. Edmunds, A., \& Morris, A. (2000). The problem of information overload in business organisations: a review of the literature. International journal of information management, 20(1), 17-28.

10. Figueira, Á., \& Oliveira, L. (2017). The current state of fake news: challenges and opportunities. Procedia Computer Science, 121, 817-825.

11. Fischer, P., S. Schulz-Hardt, and D. Frey. 2008. Selective exposure and information quantity: How different information quantities moderate decision maker's preference for (p. 544) consistent and inconsistent information. Journal of Personality and Social Psychology, 94: 231-244.

12. Farooq, A., Laato, S., \& Islam, A. N. (2020). Impact of online information on self-isolation intention during the COVID-19 pandemic: cross-sectional study. Journal of medical Internet research, 22(5), e19128.

13. Friedman, B. (1996). Value-sensitive design. interactions, 3(6), 16-23.

14. Guo, B., Ding, Y., Yao, L., Liang, Y., \& Yu, Z. (2019). The future of misinformation detection: new perspectives and trends. arXiv preprint arXiv:1909.03654.

15. Gabielkov, M., Ramachandran, A., Chaintreau, A., \& Legout, A. (2016, June). Social clicks: What and who gets read on Twitter?. In Proceedings of the 2016 ACM SIGMETRICS international conference on measurement and modeling of computer science (pp. 179-192).

16. Graf, J., \& Aday, S. (2008). Selective attention to online political information. Journal of Broadcasting \& Electronic Media, 52(1), 86-100.

17. Guo, B., Ding, Y., Yao, L., Liang, Y., \& Yu, Z. (2019). The future of misinformation detection: new perspectives and trends. arXiv preprint arXiv:1909.03654.

18. Garrett, R. K., \& Weeks, B. E. (2017). Epistemic beliefs' role in promoting misperceptions and conspiracist ideation. PloS one, 12(9), e0184733. 
19. Hodas, N. O., \& Lerman, K. (2012, September). How visibility and divided attention constrain social contagion. In 2012 International Conference on Privacy, Security, Risk and Trust and 2012 International Confernece on Social Computing (pp. 249-257). IEEE.

20. Johnston, D. W., Piatti, M., \& Torgler, B. (2013). Citation success over time: Theory or empirics?. Scientometrics, 95(3), 1023-1029.

21. Jacko, J. A. (Ed.). (2012). Human computer interaction handbook: Fundamentals, evolving technologies, and emerging applications. CRC press.

22. Knobloch-Westerwick, S., \& Meng, J. (2009). Looking the other way: Selective exposure to attitude-consistent and counterattitudinal political information. Communication Research, 36(3), 426-448.

23. Lee, K., \& Choo, H. (2013). A critical review of selective attention: an interdisciplinary perspective. Artificial Intelligence Review, 40(1), 27-50.

24. Laato, S., Islam, A. N., Islam, M. N., \& Whelan, E. (2020). What drives unverified information sharing and cyberchondria during the COVID-19 pandemic?. European Journal of Information Systems, 29(3), 288-305.

25. Lanham, R. A. (2006). The economics of attention: Style and substance in the age of information. University of Chicago Press.

26. Mohd Salihan Ab Rahman, Nazlena Mohamad Ali, Masnizah Mohd. 2017. Comelgetz Prototype in Learning Prayers among Children. Asia-Pacific Journal of Information Technology and Multimedia. 6 (1): $115-125$.

27. McLeod, S. (2008). Cognitive dissonance. Simply psychology, 31(1), 2-7.

28. Pieters, R., \& Warlop, L. (1999). Visual attention during brand choice: The impact of time pressure and task motivation. International Journal of research in Marketing, 16(1), 1-16

29. Posner, M. I., Snyder, C. R., \& Davidson, B. J. (1980). Attention and the detection of signals. Journal of experimental psychology: General, 109(2), 160.

30. Spence, C., Lloyd, D., McGlone, F., Nicholls, M. E., \& Driver, J. (2000). Inhibition of return is supramodal: a demonstration between all possible pairings of vision, touch, and audition. Experimental Brain Research, 134(1), 42-48.

31. Singh, L., Bansal, S., Bode, L., Budak, C., Chi, G., Kawintiranon, K., ... \& Wang, Y. (2020). A first look at COVID-19 information and misinformation sharing on Twitter. arXiv preprint arXiv:2003.13907.

32. Taber, C. S., \& Lodge, M. (2006). Motivated skepticism in the evaluation of political beliefs. American journal of political science, 50(3), 755-769.

33. Tukachinsky, R., \& Stever, G. (2019). Theorizing development of parasocial engagement Communication Theory, 29(3), 297-318.

34. Vosoughi, S., Roy, D., \& Aral, S. (2018). The spread of true and false news online. Science, 359(6380), 1146-1151.

35. Walter, N., \& Tukachinsky, R. (2020). A meta-analytic examination of the continued influence of misinformation in the face of correction: How powerful is it, why does it happen, and how to stop it?. Communication Research, 47(2), 155-177.

36. Yantis, S. (2000). Goal-directed and stimulus-driven determinants of attentional control. Attention and performance, 18, 73-103.

37. Zizlsperger, L., Sauvigny, T., \& Haarmeier, T. (2012). Selective attention increases choice certainty in human decision making. PLoS One, 7(7), e41136. 